To the Editors:

\title{
Drug induced megaloblastic anaemia with abnormal skin pigmentation
}

We report a case of drug induced megaloblastic anaemia occurring with pyrimethamine induced abnormal skin pigmentation, a very rare presentation.

A 19-year old boy with cervical lymphadenopathy, no constitutional symptoms, a positive lymph node biopsy and high levels of IgG for toxoplasma had a 6-week course of cotrimoxazole and pyrimethamine. He developed vomiting, abdominal pain, diarrhoea, loss of appetite, loss of weight and a grey discolouration of his face, axillae, hands and feet. Addison's disease had been excluded by appropriate laboratory investigation at this hospital. As there was worsening of his condition even after three weeks of treatment the patient sought admission to our unit.

On admission to our unit he was pale, wasted and had hair loss. Although the features were suggestive of Addison's disease, considering his history, a drug induced disorder needed to be excluded. Investigation revealed pancytopaenia with a reticulocyte count of $1 \%$ (1). Bone marrow biopsy confirmed megaloblastic erythropoiesis. The red cell folate serum level was $146 \mathrm{ng} / \mathrm{ml}$ (reference range 186-645), with a normal serum B12. HIV screening was negative and CD4, CD8 counts were normal. Other biochemical and haematological investigations were normal. A diagnosis of drug induced megaloblastic anaemia due to folate deficiency with abnormal skin pigmentation due to pyrimethamine was made. Cotrimoxazole and pyrimethamine were withdrawn and the patient was treated with folic acid. Addison's disease was excluded by the results of biochemical investigations.

Signs of clinical improvement were quickly evident. Treatment response was assessed after one week by the reticulocyte count $(7 \%)$, and thereafter by repeated blood counts, After 6 weeks, his blood counts became normal, but the skin pigmentation (Figures 1 and 2) lasted for 5 months before gradually disappearing.

Sulphonamides, trimethoprim and pyrimethamine (2) are known to inhibit folic acid synthesis, effecting the production of purines and DNA, and suppressing rapidly proliferating cells such as epithelium and bone marrow. This results in hair loss, diarrhoea, vomiting, abdominal pain and megaloblastic anaemia. Skin pigmentation is also seen rarely in folate deficiency, but the distribution of pigmentation in this case and the time taken for its disappearance is more suggestive of a side effect of pyrimethamine.

Accepted indications for active intervention in toxoplasmosis include chorioretinits, active toxoplasmosis in immunodeficiency, infected neonates and pregnant women after the first trimester. The treatment is a combination of pyrimethamine and sulphadiazine and management includes regular follow up with blood counts. This case shows the importance of taking a good drug history, and adherence to prescribed indications for intervention (3) and follow up for possible side effects when potentially toxic drugs are used.

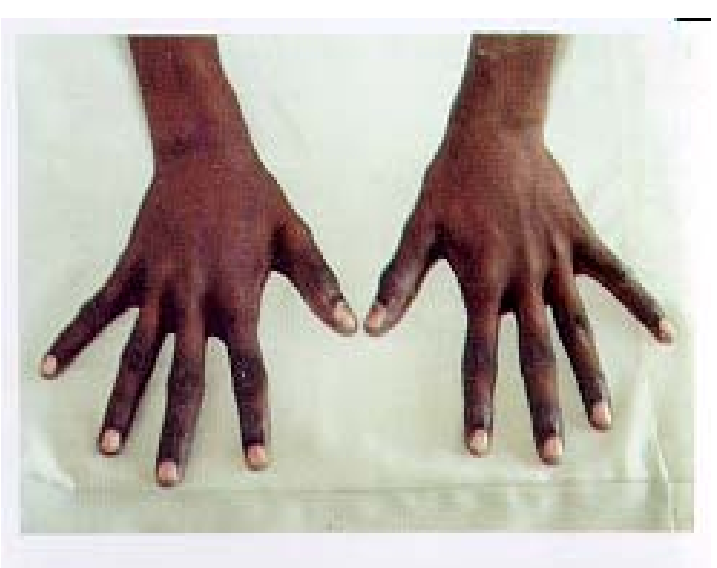

Figure 1. Skin pigmentation on admission to our unit.

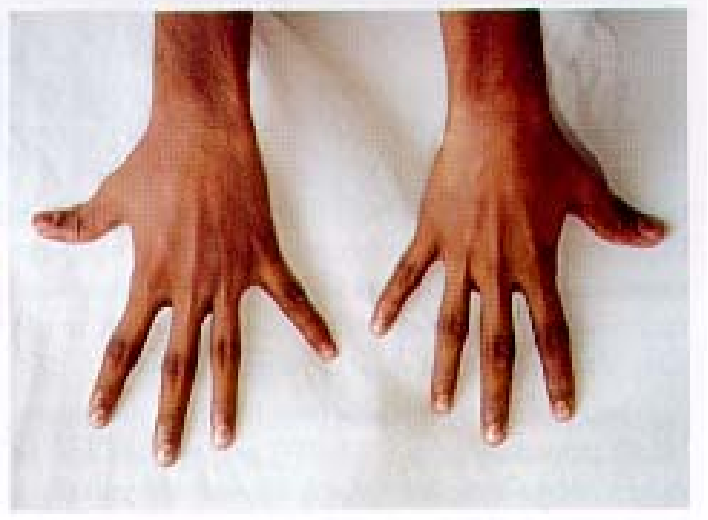

Figure 2. Photograph after 5 months of folic acid and stopping pyrimethamine and cotrimoxazole.

\section{References}

1. Blackwell EA, Hawson GAT. Acute pancytopaenia due to megaloblastic arrest in association with cotrimoxazole. Medical Journal of Australia 1978; 2: 38-41.

2. Nanthan L, Kobrinsky MD, Norma KC. Acute megaloblastic anaemia induced by high dose of trimethoprim and sulphamethoxazole. Annals of Internal Medicine 1981; 94: 780-1.

3. Toxoplasma, drugs used in parasitic disease, WHO model prescribing information, 2nd edition, WHO Geneva, 1995: 75-78.

D U S Bulugahapitiya, Registrar, Chintaka de Silva, Physician, and R Sutharson, Senior Registrar, Sri Jayawardenepura General Hospital, Nugegoda, Sri Lanka. (Received 5 December 2001, accepted 9 March 2002. Corresponding author DUSB, email: bulla@eureka.lk telephone+941669361). 